\title{
A platform for storage and analysis of results of genome-wide association studies of sheep
}

\author{
Alexander S. Zlobin \\ Kurchatov Genomic Center \\ of ICG SB RAS \\ Novosibirsk, Russia \\ zlobin@bionet.nsc.ru
}

Natalya A. Volokova

L.K. Ernst Federal Science Center for Animal Husbandry,

Dubrovitsy, Moscow Region, Russia

Yakov A. Tsepilov

Laboratory of Recombination and Segregation Analysis

Institute of Cytology and Genetics

SB RAS, Novosibirsk, Russia

\author{
Anatoliy V. Kirichenko \\ Laboratory of Recombination and \\ Segregation Analysis \\ Institute of Cytology and Genetics \\ SB RAS, Novosibirsk, Russia
}

Pavel M. Borodin

L.K. Ernst Federal Science Center for

Animal Husbandry,

Dubrovitsy, Moscow Region, Russia

Yurii S. Aulchenko

Kurchatov Genomic Center of ICG

SB RAS, Novosibirsk, Russia
Tatyana I. Shashkova

Laboratory of Theoretical and Applied Functional Genomics

Novosibirsk State University

Novosibirsk, Russia

\author{
Lennart C. Karssen \\ PolyOmica \\ 's-Hertogenbosch, the Netherlands
}

\begin{abstract}
We developed a prototype of a platform for aggregation, storage and analysis of the results of genomewide association scans of different economically important ovine traits.
\end{abstract}

Keywords - genome-wide association study; big data; genomic selection; sheep; $Q T L$

\section{Introduction}

Sheep breeding is one of the most important branches of agriculture. Currently, the most popular product of sheep breeding is mutton. For this reason, the modern market demands the breeds or breed types of sheep characterized by good viability, high growth rate, good meat qualities, ability to effectively use feed. The solution of the problem of production of pedigree sheep of competitive quality (both on the domestic and world markets) can only be achieved through the shift of selection to a qualitatively new level - from selection by phenotype to the selection at the gene and genomic level. Genome-wide association studies (GWAS) is a modern, agnostic, data-driven method for identifying QTL associated with different traits. The results of genome-wide association studies (RGWAS) are an example of big data that further can be used to build models of marker-assisted and genomic selection and identification of candidate targets for genomic editing. However, the extraction of new knowledge from big data requires its aggregation and the construction of infrastructure for its storage, access and processing. In the context of RGWAS for economically important traits o f ovines, this highly relevant problem has not been solved yet.

Aim: to implement a prototype of a platform for aggregation, storage and analysis of the results of genome-wide association scans (RGWAS) of different ovine traits.

\section{Materials and methods}

For searching and filtering papers we used PubMed (https://www.ncbi.nlm.nih.gov/pubmed/) and Google Scholar (http://scholar.google.com). We restricted our search to papers published in English, in peer-reviewed journals since 2013. We chose 2013 as a year when the first biggest GWAS $(n>200)$ for sheep was published [1]. We used a few keyword combinations which included word related to the organism (sheep/ovine/Ovis aires) and word related to the type of study (GWAS). For example, one of the keyword combinations was "sheep GWAS". For each keyword combination, we manually screened and selected for further consideration top 20 most relevant papers.
Further, for each study, we have checked the information about the full RGWAS availability. If it was publicly available we have downloaded it. In other cases, we have collected the emails of corresponding authors and contacted them with the request for the full RGWAS.

We used GWAS-MAP platform [4] to consolidate and store ovine RGWAS, and the PheLiGe web-interface to present and visualize data. First, RGWAS are converted into useful format and check correspondence between rs id and chromosome position based on the reference, and harmonize effect alleles. Second, data should pass quality control (QC). QC contains three steps: comparing allele frequency between RGWAS and reference data, check effect size distribution, and check that reported p-values correspond to z-scores. If QC is fine, RGWAS are saved in the database. For data storage and manipulations on GWAS-MAP platform we use two database management systems, ClickHouse, to store harmonized RGWAS and PostgreSQL for metadata storage. We also present web-interface PheLige to provide user-friendly access to the database and visualize data by interactive plots.

\section{Results}

Using 3 different keywords combination (sheep GWAS, ovine GWAS, ovis aries GWAS) we found 86 relevant papers (including duplicates). Next, all papers were manually curated and filtered by relevance. We selected the most relevant studies that led to the final list of 38 publications. About half of the papers described traits related to meat productivity and growth. Other traits were related to wool production, milk production, and some body composition traits (such as root, fat tail, ear size, etc.). Only two studies made the full RGWAS publically available $[2,3]$. For other studies we have contacted corresponding authors for the data access.

Next, we made the LD reference for sheep based on 96 animals (18 Romanov breed sheep, 6 Katahdin breed sheep, 10 argalis, 48 F1 hybrids Romanov sheep and argali, 14 F1 hybrids Romanov sheep and Katahdin sheep) that were genotyped by Infinium ${ }^{\circledR}$ HD SNP Bead-Chip (Illumina Inc., San Diego, CA, USA). The LD reference contains information about 523578 nonmonomorphic SNP.

We have implemented a prototype of a platform for aggregation, storage and analysis of RGWAS of ovine traits. The platform is accessible via public (web-based) and private (ssh- 
based) interfaces. At the moment, the database contains 21 millions of associations.

\section{ACKNOWLEDGMENT}

$\mathrm{AZ}$ analysed the literature, collected the data, performed quality control and developed the content of the database of the results of genome-wide association studies of ovine traits. The work of AZ was funded by the Kurchatov Genomics Center of IC\&G (075-15-2019-1662). The work of AK, who was in charge of the software setup, was funded by the Federal Agency of Scientific Organizations via the Institute of Cytology and Genetics SB RAS (project \#0324-2019-0040-C-01). The work of TS on GWAS-MAP was funded by PolyKnomics BV and by the Novosibirsk State University via Russian Ministry of Science and Education under the 5-100 Excellence Programme. The work of LK on GWAS-MAP was funded by PolyKnomics BV. The generation of data data for the linkage disequilibrium reference was supported by Russian Science Foundation \#18-16-00079 (YT and VIZh). YA and YT jointly supervised the study. The work of YA was funded by the Kurchatov Genomics Center of IC\&G. All authors contributed to writing of the manuscript.

\section{REFERENCES}

[1] Zhang, L., Liu, J., Zhao, F., Ren, H., Xu, L., Lu, J., Zhang, S., Zhang, X., Wei, C., Lu, G., Zheng, Y. and Du, L.: Genome-wide association studies for growth and meat production traits in sheep., PLoS One, 8(6), e66569, doi:10.1371/journal.pone.0066569, 2013.

[2] Bolormaa, S., Hayes, B. J., van der Werf, J. H. J., Pethick, D., Goddard, M. E. and Daetwyler, H. D.: Detailed phenotyping identifies genes with pleiotropic effects on body composition, BMC Genomics, 17(1), 224, doi:10.1186/s12864-016-2538-0, 2016.

[3] Di Gerlando, R., Sutera, A.M., Mastrangelo, S., Tolone, M., Portolano, B., Sottile, G., Bagnato, A., Strillacc, M.G. and Sardina M.A.: Genome-wide association study between CNVs and milk production traits in Valle del Belice sheep. PLoS ONE 14(4): e0215204. https://doi.org/10.1371/journal.pone.0215204, 2019.

[4] Gorev D.D., Shashkova T.I., Pakhomov E., "GWAS-MAP: a platform for storage and analysis of the result of thousands of genome-wide association scans", Bioinformatics of Genome Regulation and StructurelSystems Biology, The Eleventh International Conference, C. 43. 2018 